result and was rejected: "Not an oil drop" is written in the notebook. Other drops were rejected for a variety of reasons. But although Millikan indulged in some trimming, the reanalysis confirms the validity of his procedure and the accuracy of his results.

These case histories, and a few others more briefly described, provide valuable material for philosophical reflection. They strongly support the scientist's belief that his experiments tell us about the world, and that crucial experiments can be carried out. This is worth saying, particularly as some philosophies of science throw doubt on it. Thus the kuhnian account of scientific revolutions stresses that the incommensurability of the terms in competing paradigms prevents experimental comparison. Franklin, however, shows that although experiments are theory-laden they can be described in theory-neutral terms and can provide a crucial test of competing paradigms. This is illustrated by an account of such an experiment for newtonian and einsteinian mechanics, an exemplary case used by Kuhn. An historical example is Lummer and Pringsheim's experiment on the spectrum of black-body radiation that led to Planck's quantum theory.

Franklin also discusses the DuhemQuine problem, namely that it seems that a theory can never be definitely refuted because it is always possible to modify some auxiliary assumptions to bring it into line with experiment. How do we know, for example, that in the Wu parity nonconserving experiment something did not invert somewhere else to maintain parity conservation? There is no answer to this type of scepticism, but it is irrelevant to science. Scientists have developed their own ways of dealing with the problem of auxiliary assumptions, and Franklin analyses them in terms of bayesian confirmation theory. Essentially, this says that "we have degrees of belief in statements or hypotheses and that these degrees of belief obey the probability calculus". Franklin shows that they make good sense of his chosen examples of crucial experiments.

Franklin's analyses show clearly the overall reliability of experimental science, confirming and justifying the methods instinctively adopted by working scientists. Scientists seldom study the philosophy of science before embarking on their researches, and arguably this is just as well. But certainly a deeper insight into what they are doing is valuable, and this has now been provided.

Peter E. Hodgson (who began his career in physics as an experimentalist) is Head of the Nuclear Physics Theoretical Group, Nuclear Physics Laboratory, University of Oxford, Keble Road, Oxford OXI 3RH, UK.

\section{A rare discipline}

\section{James D. Thomson}

Conservation Biology: The Science of Scarcity and Diversity. Edited by Michael E. Soulé. Sinauer/Blackwell Scientific: 1986. Pp.584. Hbk \$50, £40; pbk \$27.95, $£ 23$.

Books that attend the emergence of new fields have double agendas. At one level, these 25 chapters by 45 authors provide a broad review and partial synthesis of conservation biology. But 'conservation biology', which involves the self-conscious transfer of principles from the pure realm of ecology and evolution to the applied realm of preservation and repair, is a juvenile and polymorphic science. This book will join its predecessor (Conservation Biology: An Evolutionary - Ecological Perspective, edited by M. E. Soulé and B. A. Wilcox, and published by Sinauer/Blackwell Scientific in 1980) as one of the founding documents of the field. Unlike treatises in mature fields, therefore, it shares the important responsibility of defining a discipline and determining its future character. Fortunately, it is up to the task.

That future character will be strongly influenced by resolution of the tension provocative. Unsurprisingly, they span a great range from cautious optimism to pessimism, concerning both the salvation of natural diversity and the success of conservation science. Consistent with the generally diminished expectations for a concise theoretical ecology in the face of natural complexity and idiosyncracy, the ecologists see less hope for broadly applicable management principles than do the geneticists. For example, while extracting some potentially useful generalizations about community stability, the relatively optimistic theoretician Pimm needs to hedge on several fundamental points for example, a successful species introduction could cause "no, few, or many species losses" (p. 325). Speaking as a field ecologist and a preserve manager who really has to face edge effects, Janzen offers few general prescriptions other than the apt, but rather bleak, "Know the natural history of your organisms well enough that you can both anticipate their interactions and know at what distance their populations can be perturbed by a preserve edge" (p. 303). On the other hand, Templeton, a population geneticist, shows that laboratory experiments with Drosophila can provide the insights needed to set up breeding management schemes for genetically vulnerable lizards and gazelles. Biology appears to become more predictable as one gets closer to the DNA (but cleansing a population of bad gene combinations is a much tidier problem than guaranteeing it a place to live).

Among the chapters on particular habitats, the treatments of tropical deforestation (Myers) and African desertification (Le Houéron and Gillet) are especially good - and especially depressing, because both disasters continue to be driven by human overpopulation which seems virtually unstoppable. Soulé argues convincingly, however, that scientific input of the right sort can ameliorate the damage to developing countries, and Diamond proves the point that even single individuals can make a difference.

Overall, Soulé's book not only explains conservation biology, it inspires one to do it. This brings us to the conservation of conservation biologists. With the unseemly rush towards molecular biology, the abandonment of traditional training grounds (such as botany departments) and the pervasive academic disdain for applied studies (except those that make money). will there be enough habitats in tomorrow's universities to support a viable population of conservation scientists? As Soulé puts it (p. 308), "The big question is, who will take the lead in developing a management curriculum for the 21 st century?"

James D. Thomson is an Associate Professor of Ecology and Evolution at the State University of New York. Stony Brook, New York 11794. USA. 